AGSAPPLIED MATERIALCaltech Library

Subscriber access provided by Caltech Library

\title{
Article
}

\section{DNA Damage and Apoptosis Induction in Cancer Cells by Chemically Engineered Thiolated Riboflavin Gold Nanoassembly}

Abhishek Sau, Sulagna Sanyal, Kallol Bera, Sabyasachi Sen, Amrit Krishna Mitra, Uttam Pal, Prabal Kumar Chakraborty, Sayantan Ganguly, Biswarup Satpati, Chandrima Das, and Samita Basu

ACS Appl. Mater. Interfaces, Just Accepted Manuscript • DOI: 10.1021/acsami.7b18837 • Publication Date (Web): 16 Jan 2018

Downloaded from http://pubs.acs.org on January 17, 2018

\section{Just Accepted}

"Just Accepted" manuscripts have been peer-reviewed and accepted for publication. They are posted online prior to technical editing, formatting for publication and author proofing. The American Chemical Society provides "Just Accepted" as a free service to the research community to expedite the dissemination of scientific material as soon as possible after acceptance. "Just Accepted" manuscripts appear in full in PDF format accompanied by an HTML abstract. "Just Accepted" manuscripts have been fully peer reviewed, but should not be considered the official version of record. They are accessible to all readers and citable by the Digital Object Identifier (DOI®). "Just Accepted" is an optional service offered to authors. Therefore, the "Just Accepted" Web site may not include all articles that will be published in the journal. After a manuscript is technically edited and formatted, it will be removed from the "Just Accepted" Web site and published as an ASAP article. Note that technical editing may introduce minor changes to the manuscript text and/or graphics which could affect content, and all legal disclaimers and ethical guidelines that apply to the journal pertain. ACS cannot be held responsible for errors or consequences arising from the use of information contained in these "Just Accepted" manuscripts. 


\section{Introduction}

The therapeutic applications of nanoparticles in regulating the live cell functions have become the most challenging aspects of nano-biotechnology $y^{1,2}$ and nanomedicine ${ }^{3,4}$. The gold nanoparticles (AuNPs) have received considerable attention in the biological and biomedical communities due to their potential use in diagnostic and therapeutic applications ${ }^{3,5-8}$. The AuNPs are usually conjugated with drugs to enhance the efficiency of targeted delivery, thus hold great promises in tumour therapy and drug delivery due to their ability to serve as biocompatible scaffolds for intracellular targeting ${ }^{9,10}$. Moreover, their nanoscale size allows them to enter cells and to target the different organelles i.e. endosome, nucleus and mitochondria ${ }^{11-13}$. The riboflavin (Rf) is widely distributed in human tissues in free or conjugated forms and plays an important role in formation of flavoprotein enzyme ${ }^{14,15}$. The Rf that consists of an isoalloxazin moiety and a ribityl chain, possesses mixed features of hydrophobic and hydrophilic functionalities $^{16,17}$, which generate its affinity towards different biological macromolecules including proteins and $\mathrm{DNA}^{18}$. Moreover, the $\mathbf{R f}$ receptor protein constitutes one type of tumour biomarkers due to their over expression in malignant cells of human breast and prostate cancers ${ }^{19,20}$.

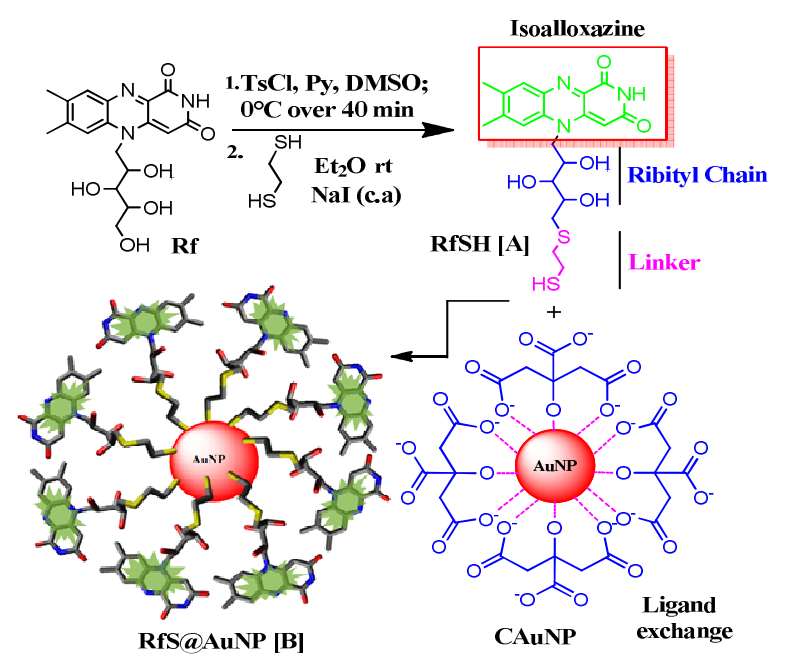

Scheme 1: Synthetic route of RfS@AuNPs

Taking advantage of the above properties of Rf and AuNPs, we have attempted to design and synthesize one of their modified conjugates, RfS@AuNPs which could facilitate significant photophysical changes due to DNA intercalation in vitro. Their smart nuclear targeted accumulation in HeLa cells can perturb the highly regulated cell cycles leading to 

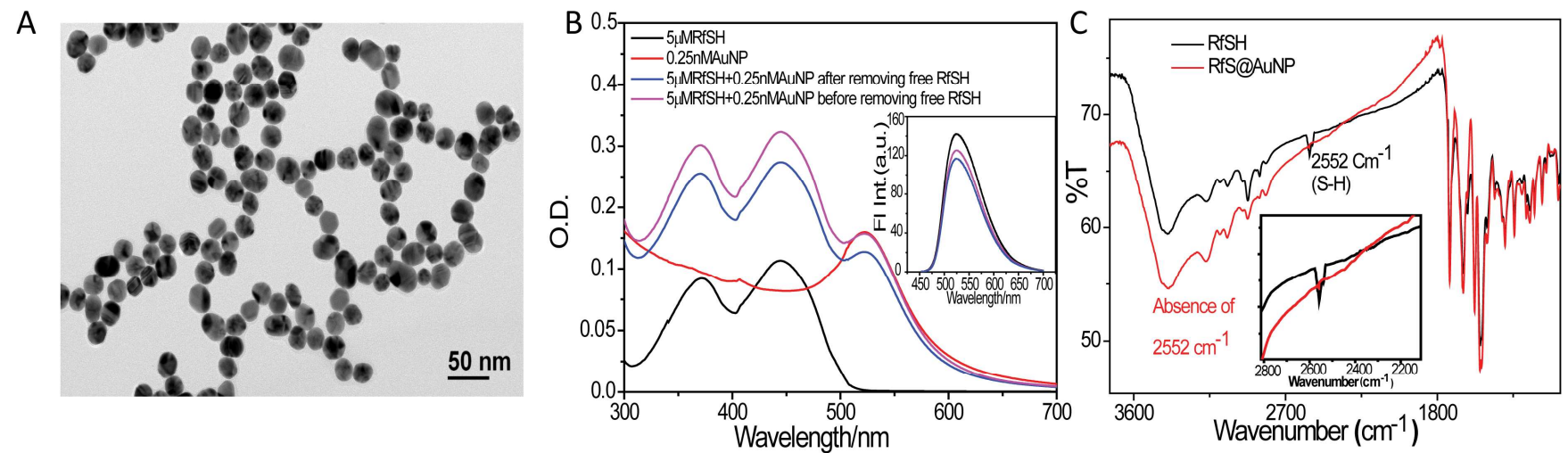

Figure 1 (A) TEM micrograph of RfS@AuNPs. (B) Absorption spectra of RfSH $\left(5 \times 10^{-6} \mathrm{M}\right)$, CAuNPs $(0.25 \mathrm{nM}$ and RfS@AuNPs (before and after removing free RfSH). Inset shows the change in fluorescence intensity of RfSH up on addition of CAuNPs and subsequent removal of free RfSH (C) IR spectra of RfSH and RfS@AuNP. Inset diagram shows the disappearance of S-H band after nano conjugation.

DNA damage and ultimate cell death. We have also attempted to elucidate the pathway of cell death by global gene expression through microarray analysis.

\section{Results and Discussion}

\section{Optical properties of RfS@AuNPs:}

The terminal hydroxyl group of ribityl chain of $\mathbf{R f}$ has been modified due to lack of suitable functional group required for the covalent attachment with citrate capped AuNPs (CAuNPs) without affecting isoalloxazine moiety. This modification is carried out by dimercaptoethane through tosylation (1) followed by substitution (2) which converts Rf to RfSH (Scheme 1). Now RfSH can easily be attached on the surface of 20-25 nm CAuNPs by ligand exchange through Au-S bond resulting in the formation of RfS@AuNPs (Scheme 1, Figure 1A, S1).

The absorption spectra of $\mathbf{R f}$ and $\mathbf{R f S H}$ show two maxima at $368 \mathrm{~nm}$ and $444 \mathrm{~nm}$ (Figure S2A) which remain more or less unaffected with increasing [CAuNPs] $(0.25-1.5 \mathrm{nM})$ in $5 \mu \mathrm{M}$ aqueous solution of RfSH (Figure 1B, S2B); hence, no significant interaction between isoaloxazine moiety and CAuNPs (TEM Figure S4B) might be inferred. Free RfSH is removed from the complex by centrifugation followed by washing and the resuspended solution is used for further experiments after sonication (Figure 1B) .The red shift of the CAuNPs Plasmon peak by $\sim 4 \mathrm{~nm}$ at higher concentration of nanoparticles is observed probably due to change in the dielectric environment via surface attachment of RfSH (Figure S2B). It has been estimated from the absorption spectra that 22800 RfSH molecules are attached with each AuNP which is much higher than that for Rf $[\sim 4680]$ (S4/Figure S3). It is now evident that RfSH has $\sim 5$ times greater binding affinity towards CAuNP compared to Rf (section S4). Again the fluorescence maximum of $\mathbf{R f}\left(\lambda_{\max }=524 \mathrm{~nm}\right)$ is very close to the absorption maximum of CAuNP $\left(\lambda_{\max }=525 \mathrm{~nm}\right)$. Therefore energy transfer may be responsible for quenching of fluorescence of $\mathbf{R f}$ with gradual increase of [CAuNPs] (section S6, Figure S5). The fluorescence lifetimes $(\tau)$ of $\mathbf{R f}$ and RfSH in aqueous medium are $4.73 \mathrm{~ns}$ and $4.69 \mathrm{~ns}$ respectively (Figure S6) which remain more or less unchanged with the addition of higher [CAuNPs] $(1 \mathrm{nM})$. This suggests that the fluorescence quenching of Rf or RfSH is a static one due to the formation of ground state complex with AuNPs. The attachment of Rf and RfSH on AuNPs surface has been verified further by FT-IR (Figure 1C) where the peak of RfSH at $2552 \mathrm{~cm}^{-1}$ disappears after the formation of RfS@AuNP. The above results indicate that the complex formed between RfSH and AuNP in ground state is quite stable and might be able to interact with the biological macro-molecules like DNA.

\section{In vitro DNA intercalation by RfS@AuNPs}

The isoalloxazine moiety of $\mathbf{R f}$ is able to intercalate DNA due to planer structure ${ }^{21,22}$. Therefore we have studied binding interaction of RfS@AuNPs with calf thymus DNA (ct-DNA) as a model system. With increasing [RfS@AuNPs] the absorption maximum of ct-DNA at $257 \mathrm{~nm}$ is blue shifted by $\sim 15 \mathrm{~nm}$ which is higher w.r.t. $\mathbf{R f}(\sim 4 \mathrm{~nm})$ as well as Rf@AuNPs $(\sim 8 \mathrm{~nm})$ (Figure 2A, S7). Upon excitation $\left(\lambda_{\text {ex }}=\right.$ $444 \mathrm{~nm}$ ) a regular increase of fluorescence intensity with the gradual addition of ct-DNA $(10-50 \mu \mathrm{M})$ is observed for a fixed [RfS@AuNPs] (Figure S8). This fluorescence enhancement occurs due to stacking interaction of isoalloxazine moiety with the base pairs of DNA, which forces them to enter inside the more constrained environment that reduces nonradiative decay. The binding constant of RfS@AuNPs with DNA $\left(\mathrm{K}_{\mathrm{a}}=\right.$ $\left.1.1 \times 10^{4} \mathrm{M}^{-1}\right)$ is $\sim 4$ times greater than that with bare RfSH $\left(\mathrm{K}_{\mathrm{a}}=2.8 \times 10^{3} \mathrm{M}^{-1}\right)$. This further supports better intercalation of RfSH inside DNA as a nano-conjugate (Figure S8C). 
The CD spectra of ct-DNA show its conformational changes upon addition of Rf, RfSH, Rf@AuNPs and RfS@AuNPs with significant alterations in the positive $\mathrm{CD}$ bands in trisbuffer $(\mathrm{pH}=7.2)$. The intensities of the characteristic bands of ct-DNA at $245 \mathrm{~nm}$ and $277 \mathrm{~nm}$ show changes with the appearance of a new positive CD band around $340 \mathrm{~nm}$ (Figure $2 \mathrm{~B})$. The intensity of negative CD band increases gradually as a consequence of excess of Rf, Rf-SH, Rf@AuNPs and RfS@AuNPs (Figure S9) in the solution suggesting the probability of occurrence of unwinding of the DNA helix ${ }^{23}$.

Moreover, the new band around $340 \mathrm{~nm}$ might be induced from RfS@AuNPs bound to DNA helix. Recently, it has been reported that a similar positive CD band is observed beyond $310 \mathrm{~nm}$ for dinuclear complexes due to selective binding of RfS@AuNPs to guanine residues of ct-DNA ${ }^{24}$. Therefore, it is worthy to mention here that these Rf, RfSH, Rf@AuNPs and RfS@AuNPs bind ct-DNA in the ground state. However, it is observed that RfS@AuNPs are much more potent intercalator among the others.

The laser flash photolysis experiments have also been performed to study the excited state interaction between ctDNA and RfS@AuNP. The triplet-triplet transient absorption spectrum of RfS@AuNPs shows peaks around $360 \mathrm{~nm}$ and $530 \mathrm{~nm}$ in tris-buffer $(\mathrm{pH}=7.2)$ (Supplementary section $\mathrm{S} 11$; Figure 2C). It is observed that with [ct-DNA] $(10,30,40 \mu \mathrm{M})$ the overall triplet absorbance of RfS@AuNP drops off along with a comparable decrease in the lifetime $(\mathrm{T})$ of the transients at $360 \mathrm{~nm}$ and $530 \mathrm{~nm}$ from $2.8 \rightarrow 2.1 \mu$ s and $2.9 \rightarrow 2 \mu \mathrm{s}$ respectively. The reduction of absorbance and lifetime of the transients may be due to (1) the inaccessibility of the chromophore i.e., isoalloxazine which is stacked within DNA micro- environment ${ }^{25}$ and (2) the formation of ground state complex between RfS@AuNPs and DNA which perturbs the overall inter system cross over processes. Hence triplet yield of RfS@AuNPs decreases. The transient state data also reflect the ground state interaction via incorporation of RfS@AuNPs within DNA microenvironment.

We have also performed stability assays of RfS@AuNPs at different $\mathrm{pH}$ (5.0, 7.2 and 8.5) employing fluorescence spectroscopy (Figure S12) which indicates that the nanoconjugate is quite stable at $\mathrm{pH} 5.0,7.2$ and 8.5 after 18 hrs which may also be true for RfS@AuNPs in lysosomal environmen ${ }^{26}$ where $\mathrm{pH}$ is around 5.0.

\section{Simulation of DNA- RfS@AuNP intercalation}

To probe the stability as well as dynamics of the RfS@AuNPs in aqueous solution and within DNA microenvironment, we have built theoretical model using Molecular Dynamics (MD). The structure of RfS@AuNPs has been modeled and shown in Figure 3A. A small gold cluster such as $\mathrm{Au}_{144}$ cluster is selected as model of AuNPs which can bind to a maximum of $60 \mathbf{R f S H}$. A larger particle of about 20 $\mathrm{nm}$ diameter is able to accommodate extremely large number of RfSH on it. Dynamics of RfSH on the gold nano surface has been probed and is shown in the video S1. The ring structures of RfSH form stacking interactions with each other to get stabilized on the AuNP surface in presence of water. $\mathbf{R f}$ is known to intercalate DNA followed by DNA strand break via generation of superoxide ${ }^{27}$. Therefore, in conjugation to AuNP, when Rf comes in contact with DNA, the planer ring of $\mathbf{R f}$ intercalates within the bases of DNA. Thus, a single molecule of RfS@AuNP via intercalation through its multiple head groups can wrap multiple strands of DNA around it. Here, Figure 3B shows the docked structure of RfSH into the DNA. MD simulation on this complex reveals that the stacking is energetically stable over time and provides a strong binding force (Video S2). If the tail of RfSH is immobilized on a surface like that of AuNP, it can immobilize DNA on that surface. An atomic model of that complex has been developed and the geometry is optimized. Figure $3 \mathrm{C}$ shows the molecular model of $\mathrm{Au}_{144}(\mathrm{RfS})_{16}$ recruiting two strands of DNA via intercalation. The RfS@AuNP bound to two strands of DNA was subjected to MD simulation for $12 \mathrm{~ns}$ in aqueous media and found to be energetically stable for the whole duration as shown in video $\mathrm{S} 3$. 

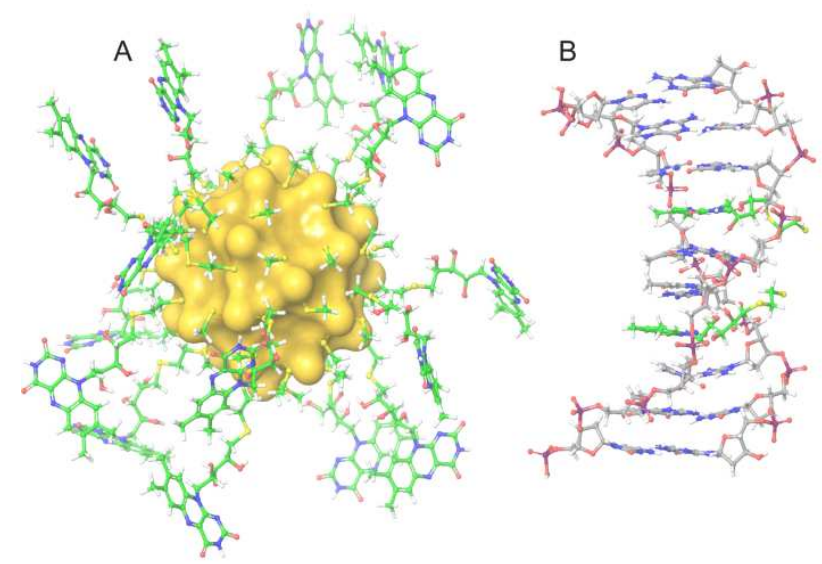

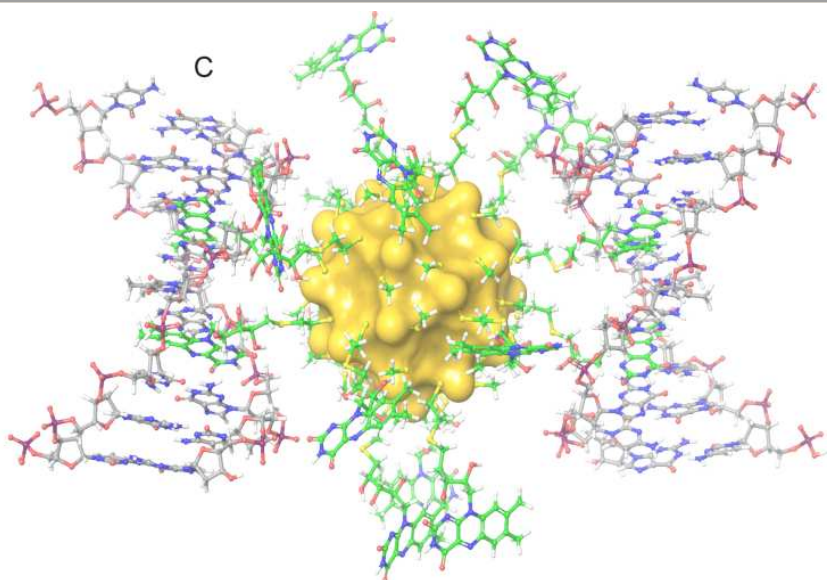

Figure 3 (A) Molecular model of $\mathrm{Au}_{144}(\mathrm{RfS})_{16}$ or model RfS@AuNPs (B) docked structure of RfSH into the DNA. (C) Molecular model of $\mathrm{Au}_{144}(\mathrm{RfS})_{16}$ recruiting two strands of DNA via intercalation.

\section{Effect of RfS@AuNPs on live cells}

We hypothesized that CAuNP functionalized with RfSH, may influence their distribution pattern in HeLa cells through the interactions of $\mathbf{R f}$ with appropriate receptors inside the cells $^{28,29}$. Further Rf was shown previously to induce apoptosis $^{30,31}$. The average size of RfS@AuNPs $(20 \mathrm{~nm}$ Figure $1 \mathrm{~A})$ is lesser than the nuclear pore size $(>90 \mathrm{~nm})^{32,33}$. Moreover these molecules are quite stable in physiological condition as shown from MD-simulation, hence can easily penetrate into the nuclei of the cells. To prove the above hypothesis, HeLa cells have been treated with Rf, RfSH, Rf@AuNPs and RfS@AuNPs and their cellular uptakes have been monitored by their auto fluorescence $\left(\lambda_{\mathrm{em}}=525 \mathrm{~nm}\right)$ in a time course dependent manner (Figure 4A-H). After $18 \mathrm{H}$, the relative fluorescence intensity analyses show the trend for nuclear uptake as RfS@AuNPs $>$ Rf@AuNPs $>$ RfSH $\cong \mathbf{R f}$ (Figure 4I). The nuclear uptake of RfS@AuNPs to the cells is markedly improved upon AuNP conjugation $[\sim 20 \%>\mathbf{R f}]$. RfS@AuNPs predominantly illustrates nuclear localization between 18-24 hrs time points (Figure 4A-D). The cells become much more rounded off in shape $12 \mathrm{H}$ onwards. Post $18 \mathrm{H}$ time point depicts cellular membrane blebbing ${ }^{34,35}$ and formation of the binucleated apoptotic bodies ${ }^{36,37}$ (Figures 4 M-Q) which are the signatures of apoptosis by RfS@AuNPs. The live cell imaging provides us with a more in-depth view of the process (Video S4). It is to be mentioned that the unconjugated RfSH, Rf and CAuNP do not show any significant change even after $24 \mathrm{H}$ treatment (Figures $4 \mathrm{~J}$, K, L).

We have quantified the nuclear and cytosolic distribution of Rf@AuNPs and RfS@AuNPs at each time point of the confocal images (Figure S11). We observe a preferential nuclear localization of RfS@AuNPs which peaks at $24 \mathrm{H}$ time point, unlike Rf@AuNPs. We have further checked the induction of specific epigenetic signature in the context of DNA damage response upon treatment with Rf@AuNPs and RfS@AuNPs. Histone H2AX is a variant of $\mathbf{H 2 A}$ histone that is phosphorylated to form $\gamma-\mathrm{H} 2 \mathrm{AX}$ in response to double strand DNA breaks ${ }^{38,39}$. Figures 5A-F show confocal images of HeLa cells in which the nuclei are stained with DAPI (blue) and anti- $\gamma$-H2AX antibodies are with red. For cells treated with 0.5 nM RfS@AuNPs, bright-red DNA damage foci
(Supplementary section S2e, Figure 5B) are observed in the nuclei in comparison to untreated cells (Figures 5D-F).

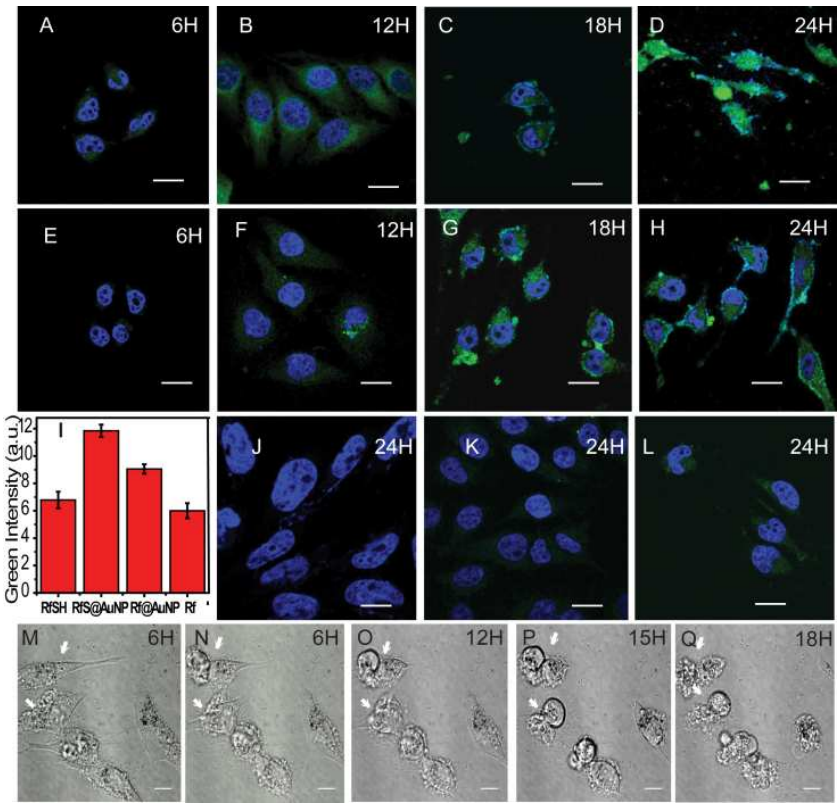

Figure 4: Real time confocal images of 0.5 nM RfS@AuNPs treated HeLa cell captured at (A) $6 \mathrm{H} \mathrm{(B)} 12 \mathrm{H} \mathrm{(C)} 18 \mathrm{H}$ (D) $24 \mathrm{H}$ (100X objective; Scale Bar represents $20 \mu \mathrm{m}$ ) and (E) $6 \mathrm{H}(\mathrm{F}) 12$ H (G) 18 H (H) $24 \mathrm{H}$ for Rf@AuNPs treated HeLa cells (Scale Bar represents $20 \mu \mathrm{m}$ ) (I) Relative green fluorescence Intensity of RfSH; Rf@AuNPs; RfS@AuNPs; Rf fluorescence in the HeLa nucleus after $12 \mathrm{H}$ treatment. (J, K, L) Real-time images of cancer cells (HeLa) at maximum time point $(24 \mathrm{H})$ after treatment of $(\mathrm{J})$ CAuNP (K) Only Rf (L) only RfSH (Scale Bar represents 20 $\mu \mathrm{m})$; Panel: $\mathrm{M}, \mathrm{N}, \mathrm{O}, \mathrm{P}, \mathrm{Q}$ are the representative time-lapse images of a HeLa cell treated with 0.5 nM RfS@AuNPs undergoing membrane blebbing and binucleated (indicated by arrow) cell formation. (The scale bar is $10 \mu \mathrm{m}$.) 
These results have been reconfirmed by immunoblotting experiments (Figure 5I). The DNA double strand break can be caused by various cellular events like replication fork collision, apoptosis, external damage and dysfunctional telomeres that may lead to phosphorylation of $\mathrm{H} 2 \mathrm{AX}^{40,41}$. Further, the flow-cytometric analysis has been carried out to monitor the ability of RfS@AuNPs to modulate cell cycles (Figure 5G, S10B). The Pre-G1 peak shows a huge increase in presence of RfS@AuNPs ( 46.4 \% of total cells) treated for $24 \mathrm{H}$ as compared to control untreated cells $(\sim 4.6 \%$ of total cells). The alteration in the percentage of cells in different stages of cell cycle is represented graphically (Figure 5G, S10). Hence most of the RfS@AuNPs treated cells are arrested at pre-G1 phase, indicating cell death ${ }^{42,43}$ more potently as compared to Rf. The DNA fragmentation in apoptotic cells are quantified by DNA ladder assay and found to be maximum in case of RfS $\boldsymbol{Q}$ AuNP treated cells (Figure 5H). The suggested cause for cell death in CAuNP-treated cells is the generation of reactive oxygen species (ROS), which is known to cause irreversible DNA damage ${ }^{44,45}$.
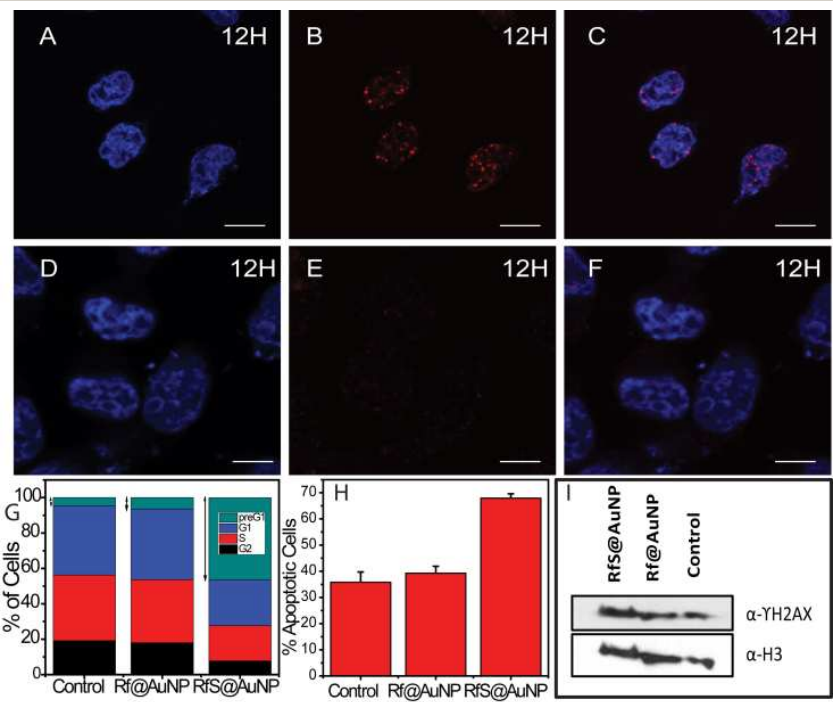

Figure 5. Immuno fluorescence staining by $\gamma-\mathrm{H} 2 \mathrm{AX}$ antibody. Confocal image of HeLa cells treated with 0.5 nM RfS@AuNPs (A) blue channel (B) red channel (C) merged image of (A) \& (B). Untreated HeLa (D) blue channel (E) red channel (F) merged image of (E) \& (F). (In 100x-objective, Scale Bar $=20 \mu \mathrm{m})$. (G) Flow-cytometric analysis showed \% of cells in each phase (cyanpreG1; blue-G1; red-S; black-G2) of the cell cycle for HeLa cells in the presence of $0.5 \mathrm{nM}$ Rf@AuNPs and RfS@AuNPs, suggesting cell death. $(\mathrm{H})$ DNA fragmentations in apoptotic cells are quantified by DNA ladder assay (I) Immuno-blotting by $\gamma$ $\mathrm{H} 2 \mathrm{AX}$ antibody. $\alpha-\mathrm{H} 3$ is used as a loading control.
Global gene expression analysis upon RfS@AuNPs treatment

Treatment of the RfS@AuNPs perturbs a large number of biological processes in the HeLa cells, including vital processes like DNA repair processes, chromatin organization, mitosis, cell cycle etc. It is well evident from the list of affected processes that the gold nano assembly leads to loss of cell viability for the cancer cells. This fact coupled with the selective targeting of the cancer cells through $\mathbf{R f}$ receptors renders a unique specificity to the gold nano assembly, and represents a potential mode of modern cancer therapy. Specific targeting and delivery of nano particle can bypass the non specific cytotoxic effect of radiation and chemotherapy, and thus, it is currently a major area of anti cancer drug designing. Our current study sheds light on a unique mode of tumor specific nano particle conjugated drug delivery and also advances our current understanding of nano particle effect on cellular processes. Selective upregulation of pro-apoptotic markers like $\mathrm{Bax}^{46}$, IL- $6^{47}$ and subsequent down regulation of anti-apoptotic markers like STAT1 ${ }^{48}$ and BCL2L12 ${ }^{49}$ (Figure 6 A-H,S13 and S14) also indicate that RfS@AuNPs induce apoptosis.

\section{CONCLUSION}

Here we have tried to design and synthesize a nanocojugate, RfS@AuNPs, which shows four times stronger binding with ct-DNA compared to Rf, RfSH and Rf@AuNPs. The MDsimulation also confirms the stability of the conjugate in aqueous medium. Further, we have shown that the accumulation of RfS@AuNPs in the nuclei of the cells may be due to the appropriate over expressed receptor present in the treated HeLa cells. Most of the cells are arrested at pre-G1 phase, inducing DNA damage and ultimately cell death. RfS@AuNP has a number of advantages in terms of synthesis and its applications in cells. The RfS@AuNP (1) can be easily prepared from the low cost vitamin-B2 just by a simple two step process, (2) has auto green fluorescence which can be easily tracked by simple confocal microscope (i.e. one-photon excitation $\lambda_{\text {ex }}=444 \mathrm{~nm}$ ), (3) can be used in low concentration as a nucleus targeting agent without attachment of any nuclear localizing signal (NLS/RGD) peptide, dendrimer with AuNPs and (4) can deliver drug molecules in the cell nucleus by chemical attachment. AuNP-conjugated drug can regulate many cellular processes in a more efficient manner as obtained from analysis of the microarray gene expression of RfS@AuNPs treated HeLa cells. RfS@AuNPs might find new applications in tumour diagnosis and cancer therapy. 

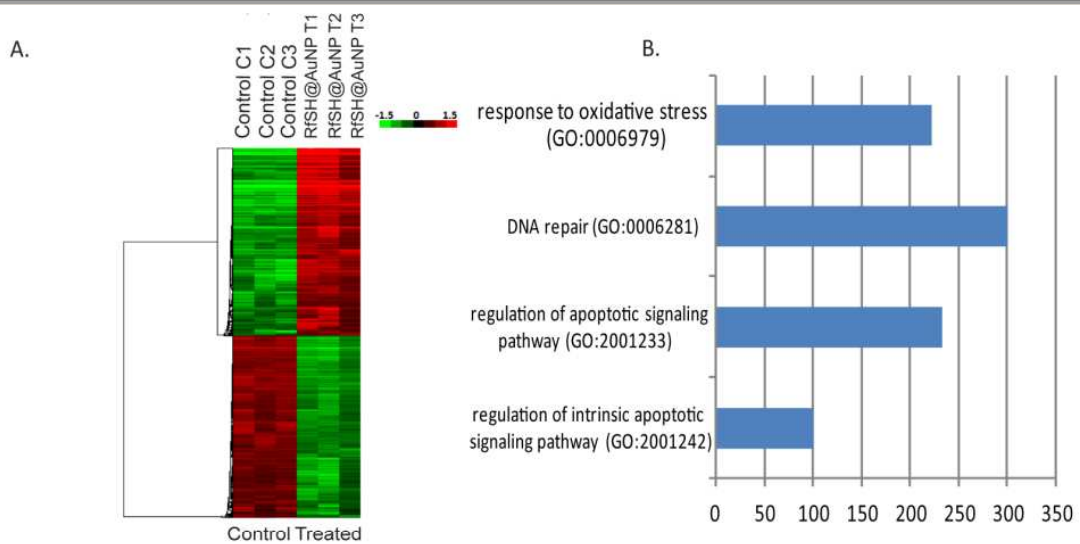

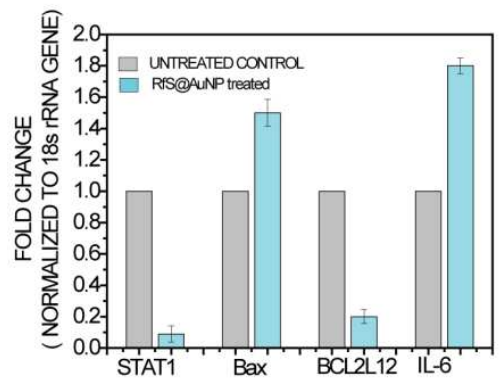

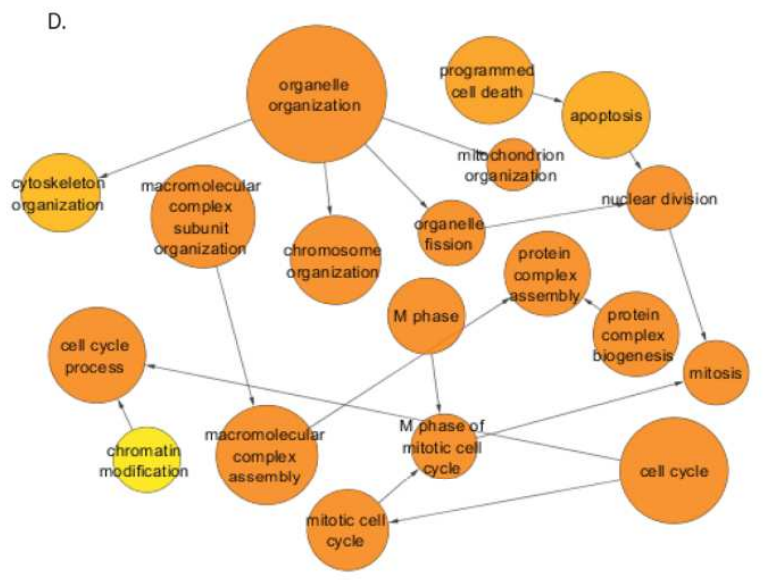
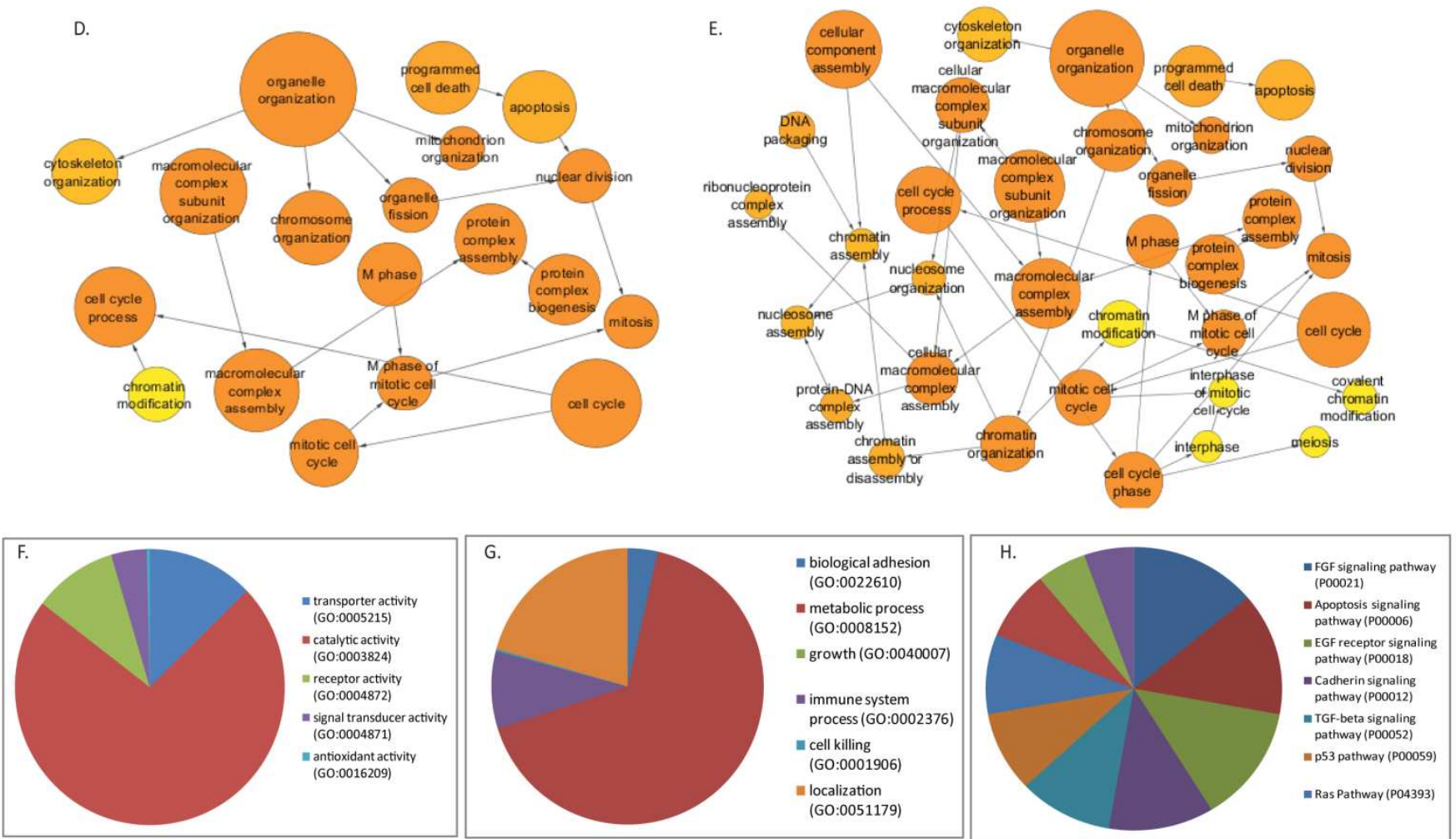

Figure 6. (A) Clustering and heat maps of expression values for differentially expressed genes. Down-regulated genes are marked in green and up-regulated genes are marked in red. From left to right first three samples are control untreated and latter three samples are $0.5 \mathrm{nM}$ RfS@AuNPs treated HeLa cells. (B) Overrepresentation of differentially expressed genes from microarray in cell survival related processes (C) Validation of candidate upregulated and down regulated genes by qRTPCR after treating the HeLa cells with RfS@AuNPs for $12 \mathrm{H}$. Normalization was done with 18srRNA and reference level was considered as 1. Each bar is an average of 3individual biological replicates. Error bars show standard deviations. (D, E) Network of differentially regulated biological processes upon nanoparticle treatment in HeLa cells. Nodes represent individual biological processes, and edges represent the connection between them. Network constructed using BiNGO tool of Cytoscape software. The colour scale represents the level of significance of the GO process (preset cut-off: $p<0.05$ ). Overrepresentation of differentially expressed genes from microarray data in following categories: F. Molecular function. G. Biological processes. H. Signaling pathways.

\section{Experimental Section}

All the chemicals and solvents required for syntheses of the thiol derivative of Riboflavin are of analytical grade. We have purchased 7,8-Dimethyl-10-[(2S,3S,4R)-2,3,4,5-tetrahy droxypentyl]benzopteridine-2,4-dione (Riboflavin), 1,2Ethanedithiol, from Aldrich Chemical Co., highly polymerized calf thymus DNA (ct-DNA) from Sisco Research Laboratory, India and tris buffer, sodium chloride and hydrochloric acid (AR) from Merck. The chemicals have been used without further purification. Triple distilled water has been used for the preparation of all the aqueous solutions. Solvents required for syntheses and spectroscopic studies have been purchased from $\mathrm{SRL}$, India and Spectrochem, India respectively. $\mathrm{HAuCl}_{4}, 3 \mathrm{H}_{2} \mathrm{O}$, trisodiumcitrate has been procurred from Sigma. Steady-state absorption and fluorescence spectra of $\mathbf{R f}$ have been recorded using JASCO V-650 absorption spectrophotometer and Spex Fluoromax-3 Spectro-fluorimeter respectively. Time-resolved emission spectra of RfS@AuNPs have been obtained using a picosecond pulsed diode laser based TCSPC fluorescence spectrometer with $\lambda$ ex $\sim 370 \mathrm{~nm}$ and MCP-PMT as a detector. Respective transient intermediates have been generated with third 
harmonic (355 nm) output of nanosecond flash photolysis set-up (Applied Photophysics) containing Nd:YAG Laser (Lab series, Model Lab 150, Spectra Physics) ${ }^{50}$. TEM measurements have been carried out using an FEI, TECNAI G2F30, S-TWIN microscope operating at $300 \mathrm{kV}$ equipped with a GATAN Orius SC1000B CCD camera. Flow cytometry has been performed on a FACS Calibur from BD Biosciences.

\section{SYNTHESES}

\section{Synthesis of RfSH.}

A $500-\mathrm{mL}$ round-bottomed three-necked flask fitted with a stirrer and a theromometer is charged with $200 \mathrm{~mL}$ of pyridine and $18.81 \mathrm{~g}(0.05 \mathrm{~mol})$ of Riboflavin (Rf) in $200 \mathrm{~mL}$ of DMSO. The solution is stirred and chilled to $0^{\circ} \mathrm{C}$ and then $9.53 \mathrm{~g}(0.05$ $\mathrm{mol}$ ) of freshly recrystallized p-toluenesulfonyl chloride is added in portions over a 20 -min period. The reaction mixture is allowed to attain room temperature and is stirred for $16 \mathrm{H}$. The mixture is re-cooled to $0^{\circ} \mathrm{C}$ and poured into $100 \mathrm{~mL}$ of concentrated hydrochloric acid in $500 \mathrm{~mL}$ of ice water. The aqueous layer is extracted with $100 \mathrm{~mL}$ of DCM and the combined organic layers are washed with two $60 \mathrm{ml}$ portions of brine, dried over magnesium sulphate and concentrated by rotary evaporation to yield $10.2 \mathrm{~g}$ of a light yellow solid of Rf-OTs. Rf-OTs (10.61g, $0.02 \mathrm{~mol})$ is dissolved in ether $(100.0 \mathrm{ml})$ by continuous stirring and then 1,2-dithiane ( $2 \mathrm{~g}, 0.02 \mathrm{~mol})$ is added in drop wise manner. The reaction mixture is stirred for 24 hours in presence of catalytic amount of $\mathrm{NaI}(0.003 \mathrm{~g}, 0.00002$ moles $)$ under room temperature. The dark yellow product is purified using column chromatography and used as RfSH

1H NMR (300 MHz, DMSO-d6) 11.34 (s, 1H, N12-H), 7.92 (s,1H, Ar-C1-H), 7.88 (s,1H, Ar-C4-H), 5.15 (s, N14-H), 4.59 (s, $\mathrm{N} 8-\mathrm{H}), 4.93,4.90,4.87,4.8(\mathrm{~m}, 1 \mathrm{H}, 1 \mathrm{H}, \mathrm{C} 18,20-\mathrm{H}), 4.80,4.79$ (d, 2H, C17-H), 4.63, 4.59 (d, 2H, C21-H), 3.47, 3.45, 3.43 (t, 1H, C19-H), 2.675, 2.671, 2.66, 2.64 (m, 2H, C24-H), 3.63 (s, 1H, 3$\mathrm{OH}), 2.58$ (s, 1H, S25-H). 2.57-2.50 (m, 2H, C23-H), $2.48(\mathrm{~s}, 3 \mathrm{H}$, C15-H), 2.40 (s, 3H, C16-H). [Figure S1 A]

${ }^{13}$ C NMR (75 MHz, DMSO-d6): 117.5(C1,C4), 135.7(C2), 136.7 (C3)150.8(C6), 146.1(C7), 132.1 (C9), 134.0(C10), 155.6 (C11), 160.0 (C13), 18.8 (C15,C16), 47.36 (C17), 63.4 (C18), 68.8(C19), 72.8 (C20), 73.6(C21), 40.3(C23), 38.2 (C24). [Figure $\mathrm{S} 1 \mathrm{~B}]$

ESI mass of RfSH: $\mathrm{m} / \mathrm{z}$ obtained for RfSH is: 454.16 (Exact mass 454.13) [Figure S1C]

Preparation of RfS@AuNPs: Aqueous solution of RfSH $\left(5 \times 10^{-4}\right.$ $\mathrm{M}$ ) is mixed with $1 \mathrm{nM}$ CAuNPs in 1:4 ratio. Resulting solution is kept for $\sim 4 \mathrm{H}$ and centrifuged (at $4000 \mathrm{rpm}$ ) for 90 minutes. This process is repeated thrice. The bottom part is collected, redispersed in water and used as RfS@AuNPs for each experimental step. Concentration used for cell treatment is $0.5 \mathrm{nM}$

Methods of MD-simulation: Coordinates of an icosahedral metallic gold cluster comprising $144 \mathrm{Au}$ atoms are obtained from the published work of Lopez-Acevedo et al. ${ }^{51}$. DNA intercalated with a thiazole orange dye is obtained from the Protein Data Bank (PDB ID: 108D). Structure of thiooxalated riboflavin (RfSH) is drawn in Schrodinger maestro molecular modeling environment. Multiple RfSH molecules are attached to the surface of the $\mathrm{Au}$ cluster through the Au-S linkage. The RfSH@AuNP complex is then subjected to the MD simulation in aqueous phase for $12 \mathrm{~ns}$. Prior to production MD structure is minimized using a previously published five step relaxation protocol ${ }^{52}$. In the DNA structure, $108 \mathrm{D}$, the ligand is exchanged for RfSH using Auto Dock 4.2 ${ }^{52}$ followed by energy minimization in Schrodinger Maestro. Two DNA strands intercalated with RfSH are then attached to the surface of $\mathrm{Au}_{144}$ via Au-S linkage. DNA bound RfS@AuNP complex is then solvated in single point charge water model and the dynamics of the system is probed for $12 \mathrm{~ns}$ in normal temperature and pressure. Restraints are added to the terminal base pairs to prevent base fraying. All the MD simulations are performed in Desmond (Academic version 2016-4) as implemented in Schrodinger Maestro ${ }^{53}$ (using previously published protocol) $)^{52}$.

\section{Microarray analysis and its validation}

Microarray analysis: Raw data are Quantile normalized and baseline transformation is done to median of all samples using Gene Spring GX 12.5 software (Agilent Technologies Inc, Santa Clara, USA).

Statistical analysis and differentially expressed genes: Differentially expressed probe sets (Genes) upon treatment in comparison to untreated cells are identified by applying Volcano Plot using a fold-change threshold of absolute fold-change greater than or equal to 1.5 and a statistically significant t-test $P$ value threshold adjusted for false discovery rate of less than 0.001 . Statistically significantly enriched transcripts with a $P$ value adjusted for false discovery rate of less than 0.05 derived using the hyper geometric distribution test corresponding to differentially expressed genes are determined using student t-test with Benjamini Hocheberg FDR test. Unsupervised hierarchical clustering of differentially expressed genes upon treatment in comparison to untreated are done using Euclidian algorithm with Centroid linkage rule to identify gene clusters whose expression levels are significantly reproduced across the replicates.

Biological pathways and gene ontology enrichment analysis: Differentially expressed gene list is subjected for biological significance analysis by GOElite tool (Ref). 21887 protein coding genes are used as the background and differentially expressed gene list is used as query. Database of Gene Ontology categories, Wiki pathways, KEGG Pathways, Pathway Commons, Pheno Ontology, Diseases, Protein Domains, Transcription factor targets and Tissue expression are configured for significant analysis. Each query list is subjected to over representation analysis against each of the above databases. A Z score and permutation or Fisher's Exact Test p-value are calculated to assess overrepresentation of enriched biological categories.

Gene regulatory network modeling: Statistically significant dysregulated genes are used as input for identification of the relevant biological processes. The output file is visualized in Cytoscape V 2.8 in the form of connections along with modeling the network. Genes are subjected to color codes depending on their statistical significance and the major biological processes are highlighted to identify biological pathways. DAVID ${ }^{54}$ and PANTHER $^{55}$ classification tools are also used to ascertain the functional classification of the Differentially Expressed genes.

RNA isolation and qRT PCR: Total cellular RNA is extracted using TRIZOL reagent (Invitrogen) as per manufacturer's instructions and RNA quantity as well as quality is determined using Nanodrop spectrophotometer (NanoDrop Technologies, Wilmington, DE). The reverse transcription is performed with revertaid first strand cDNA synthesis kit (Thermo Scientific). Primer sequences used in the assay have been provided in the supplementary section. Four genes are chosen for validation of the microarray data: STAT1, BAX, BCL2L12 and IL-6. 


\section{ASSOCIATED CONTENT}

Supporting Information. It contains details of synthesis and characterisation of RfSH and RfS@AuNPs. It also contains the Figures S1-S14 and Videos S1-S4. "This material is available free of charge via the Internet at http://pubs.acs.org."

\section{AUTHOR INFORMATION}

\section{Corresponding Author}

*samita.basu@saha.ac.in

*chandrima.das@saha.ac.in

Present Addresses

KB: Division of Biology and Biological Engineering, California Institute of Technology, Pasadena, CA 91125

\section{Author Contributions \\ ${ }^{\$}$ These authors contributed equally.}

\section{ACKNOWLEDGMENT}

We sincerely thank S. Haldar, R. Modok, Y. Sikdar, C. Sengupta, P. Mitra, S. Das Chakraborty and M. Bhattacharyya for their constant support and help. We acknowledge financial support from UGC (A. S.; F2-32/1998 (SA-1), DBT- Government of India, Ramalingaswami fellowship and BARD project: DAE at Saha Institute of Nuclear Physics..

\section{REFERENCES}

(1) Ndukaife, J. C.; Kildishev, A. V.; Nnanna, A. G. A.; Shalaev, V. M.; Wereley, S. T.; Boltasseva, A. Long-range and rapid transport of individual nano-objects by a hybrid electrothermoplasmonic nanotweezer. Nature nanotechnology 2016, 11, 53.

(2) Zhao, H.; Sen, S.; Udayabhaskararao, T.; Sawczyk, M.; Kucanda, K.; Manna, D.; Kundu, P. K.; Lee, J.-W.; Král, P.; Klajn, R. Reversible trapping and reaction acceleration within dynamically self-assembling nanoflasks. Nature nanotechnology 2016, 11, 82.

(3) Chen, G.; Roy, I.; Yang, C.; Prasad, P. N Nanochemistry and nanomedicine for nanoparticle-based diagnostics and therapy. Chemical reviews 2016, 116, 2826-2885.

(4) Bishop, K. J. Hierarchical Self-Assembly for Nanomedicine. Angewandte Chemie International Edition 2016, $55,1598-1600$.

(5) Abadeer, N. S.; Murphy, C. J. Recent progress in cancer thermal therapy using gold nanoparticles. The Journal of Physical Chemistry C 2016, 120, 4691-4716.

(6) Li, Z.; Huang, H.; Tang, S.; Li, Y.; Yu, X.-F.; Wang, H.; Li, P.; Sun, Z.; Zhang, H.; Liu, C. Small gold nanorods laden macrophages for enhanced tumor coverage in photothermal therapy. Biomaterials 2016, 74, 144-154.

(7) Aioub, M.; El-Sayed, M. A. A real-time surface enhanced raman spectroscopy study of plasmonic photothermal cell death using targeted gold nanoparticles. Journal of the American Chemical Society 2016, 138, 1258-1264.

(8) Baek, S.; Singh, R. K.; Kim, T.-H.; Seo, J.-w.; Shin, U. S.; Chrzanowski, W.; Kim, H.-W. Triple Hit with Drug Carriers: $\mathrm{pH}$-and Temperature-Responsive Theranostics for Multimodal Chemo-and Photothermal Therapy and Diagnostic Applications. ACS applied materials \& interfaces 2016, 8, 89678979.

(9) Espinosa, A.; Di Corato, R.; Kolosnjaj-Tabi, J.; Flaud, P.; Pellegrino, T.; Wilhelm, C. Duality of iron oxide nanoparticles in cancer therapy: amplification of heating efficiency by magnetic hyperthermia and photothermal bimodal treatment. ACS nano 2016, 10, 2436-2446.

(10) Sykes, E. A.; Dai, Q.; Sarsons, C. D.; Chen, J.; Rocheleau, J. V.; Hwang, D. M.; Zheng, G.; Cramb, D. T.; Rinker, K. D.; Chan, W. C. Tailoring nanoparticle designs to target cancer based on tumor pathophysiology. Proceedings of the National Academy of Sciences 2016, 113, E1142-E1151.

(11) Bera, K.; Baksi, S.; Nag, M.; Bera, S. C.; Mukhopadhyay, D.; Basak, S. A multicolor fluorescent peptidenanoparticle scaffold: real time uptake and distribution in neuronal cells. New Journal of Chemistry 2014, 38, 2739-2743.

(12) Brandenberger, C.; Mühlfeld, C.; Ali, Z.; Lenz, A. G.; Schmid, O.; Parak, W. J.; Gehr, P.; Rothen-Rutishauser, B. Quantitative Evaluation of Cellular Uptake and Trafficking of Plain and Polyethylene Glycol-Coated Gold Nanoparticles. Small 2010, 6, 1669-1678.

(13) Wang, L.; Liu, Y.; Li, W.; Jiang, X.; Ji, Y.; Wu, X.; Xu, L.; Qiu, Y.; Zhao, K.; Wei, T. Selective targeting of gold nanorods at the mitochondria of cancer cells: implications for cancer therapy. Nano letters 2010, 11, 772-780.

(14) Tan, S. L.; Webster, R. D. Electrochemically induced chemically reversible proton-coupled electron transfer reactions of riboflavin (vitamin B2). Journal of the American Chemical Society 2012, 134, 5954-5964.

(15) Mantovani, S. M.; Moore, B. S. Flavin-linked oxidase catalyzes pyrrolizine formation of dichloropyrrolecontaining polyketide extender unit in chlorizidine A. Journal of the American Chemical Society 2013, 135, 18032-18035.

(16) Nandwana, V.; Samuel, I.; Cooke, G.; Rotello, V. M. Aromatic stacking interactions in flavin model systems. Accounts of chemical research 2012, 46, 1000-1009.

(17) Legrand, Y.-M.; Gray, M.; Cooke, G.; Rotello, V. M. Model systems for flavoenzyme activity: relationships between cofactor structure, binding and redox properties. Journal of the American Chemical Society 2003, 125 , 15789-15795.

(18) Sumathi, C.; Muthukumaran, P.; Radhakrishnan, S.; Ravi, G.; Wilson, J. Riboflavin detection by $\alpha-$ Fe 2 O 3/MWCNT/AuNPs-based composite and a study of the interaction of riboflavin with DNA. RSC Advances 2015, 5, 17888-17896.

(19) Shigemizu, D.; Hu, Z.; Hung, J.-H.; Huang, C.-L.; Wang, Y.; DeLisi, C. Using functional signatures to identify repositioned drugs for breast, myelogenous leukemia and prostate cancer. PLoS Comput Biol 2012, 8, e1002347.

(20) Thomas, T. P.; Choi, S. K.; Li, M.-H.; Kotlyar, A.; Baker, J. R. Design of riboflavin-presenting PAMAM dendrimers as a new nanoplatform for cancer-targeted delivery. Bioorganic \& medicinal chemistry letters 2010, 20, 5191-5194.

(21) Sankaran, N.; Nishizawa, S.; Seino, T.; Yoshimoto, K.; Teramae, N. Abasic-Site-Containing Oligodeoxynucleotides as Aptamers for Riboflavin. Angewandte Chemie International Edition 2006, 45, 1563-1568.

(22) Beztsinna, N.; Solé, M.; Taib, N.; Bestel, I. Bioengineered riboflavin in nanotechnology. Biomaterials 2016, $80,121-133$

(23) Long, Y. F.; Liao, Q. G.; Huang, C. Z.; Ling, J.; Li, Y. F. Conformational Change Detection of DNA with the Fluorogenic Reagent of o-Phthalaldehyde- $\beta$-Mercaptoethanol. The Journal of Physical Chemistry B 2008, 112, 1783-1788.

(24) Nakabayashi, Y.; Inada, H.; Minoura, Y.; Iwamoto, N.; Yamauchi, O. Effects of flexible bridging ligands on DNA-binding of dinuclear ruthenium (II)-2, 2'-bipyridine complexes. Inorganica Chimica Acta 2009, 362, 869-877. 
(25) Pokorny, R.; Klar, T.; Hennecke, U.; Carell, T.; Batschauer, A.; Essen, L.-O. Recognition and repair of UV lesions in loop structures of duplex DNA by DASH-type cryptochrome. Proceedings of the National Academy of Sciences 2008, 105, 21023-21027.

(26) Poole, B.; Ohkuma, S. Effect of weak bases on the intralysosomal $\mathrm{pH}$ in mouse peritoneal macrophages. The Journal of cell biology 1981, 90, 665-669.

(27) Ly, D.; Kan, Y.; Armitage, B.; Schuster, G. B. Cleavage of DNA by irradiation of substituted anthraquinones: intercalation promotes electron transfer and efficient reaction at GG steps. Journal of the American Chemical Society 1996, 118, 8747-8748.

(28) Leistra, A.; Witte, A.; Choi, S. K.; Sinniah, K. An Atomic Force Microscopy Study of Riboflavin Receptor Targeting Nanoparticles. Biophysical Journal 2013, 104, 556a557a.

(29) Yazdanpanah, B.; Wiegmann, K.; Tchikov, V.; Krut, O.; Pongratz, C.; Schramm, M.; Kleinridders, A.; Wunderlich, T.; Kashkar, H.; Utermöhlen, O. Riboflavin kinase couples TNF receptor 1 to NADPH oxidase. Nature 2009, 460, $1159-1163$

(30) Wollensak, G.; Spoerl, E.; Reber, F.; Seiler, T. Keratocyte cytotoxicity of riboflavin/UVA-treatment in vitro. Eye 2004, 18, 718-722.

(31) de Souza Queiroz, K. C.; Zambuzzi, W. F.; de Souza, A. C. S.; da Silva, R. A.; Machado, D.; Justo, G. Z.; Carvalho, H. F.; Peppelenbosch, M. P.; Ferreira, C. V. A possible anti-proliferative and anti-metastatic effect of irradiated riboflavin in solid tumours. Cancer Letters 2007, 258, 126-134.

(32) Winey, M.; Yarar, D.; Giddings, T. H.; Mastronarde, D. N. Nuclear pore complex number and distribution throughout theSaccharomyces cerevisiae cell cycle by three-dimensional reconstruction from electron micrographs of nuclear envelopes. Molecular Biology of the Cell 1997, 8, 21192132.

(33) Selvi, B. R.; Jagadeesan, D.; Suma, B.; Nagashankar, G.; Arif, M.; Balasubramanyam, K.; Eswaramoorthy, M.; Kundu, T. K. Intrinsically fluorescent carbon nanospheres as a nuclear targeting vector: delivery of membraneimpermeable molecule to modulate gene expression in vivo. Nano letters 2008, 8, 3182-3188.

(34) Mills, J. C.; Stone, N. L.; Erhardt, J.; Pittman, R. N. Apoptotic membrane blebbing is regulated by myosin light chain phosphorylation. The Journal of cell biology 1998, 140, 627-636.

(35) Coleman, M. L.; Sahai, E. A.; Yeo, M.; Bosch, M.; Dewar, A.; Olson, M. F. Membrane blebbing during apoptosis results from caspase-mediated activation of ROCK I. Nature cell biology 2001, 3, 339-345.

(36) Cook, P. J.; Ju, B. G.; Telese, F.; Wang, X.; Glass, C. K.; Rosenfeld, M. G. Tyrosine dephosphorylation of H2AX modulates apoptosis and survival decisions. Nature 2009, $458,591-596$

(37) Gregory, C. D. Cell biology: the disassembly of death. Nature 2014, 507, 312-313.

(38) di Fagagna, F. d. A.; Reaper, P. M.; ClayFarrace, L.; Fiegler, H.; Carr, P.; von Zglinicki, T.; Saretzki, G.; Carter, N. P.; Jackson, S. P. A DNA damage checkpoint response in telomere-initiated senescence. Nature 2003, 426, 194-198.

(39) Bartkova, J.; Hořejší, Z.; Koed, K.; Krämer, A.; Tort, F.; Zieger, K.; Guldberg, P.; Sehested, M.; Nesland, J. M.; Lukas, C. DNA damage response as a candidate anti-cancer barrier in early human tumorigenesis. Nature 2005, 434, 864-870.

(40) Löbrich, M.; Rief, N.; Kühne, M.; Heckmann, M.; Fleckenstein, J.; Rübe, C.; Uder, M. In vivo formation and repair of DNA double-strand breaks after computed tomography examinations. Proceedings of the National Academy of Sciences of the United States of America 2005, 102, 8984-8989.

(41) Petersen, S.; Casellas, R.; Reina-San-Martin, B.; Chen, H. T.; Difilippantonio, M. J.; Wilson, P. C.; Hanitsch, L.; Celeste, A.; Muramatsu, M.; Pilch, D. R. AID is required to initiate $\mathrm{Nbs} 1 / \gamma-\mathrm{H} 2 \mathrm{AX}$ focus formation and mutations at sites of class switching. Nature 2001, 414, 660-665.

(42) Varmark, H.; Sparks, C. A.; Nordberg, J. J.; Koppetsch, B. S.; Theurkauf, W. E. DNA damage-induced cell death is enhanced by progression through mitosis. Cell Cycle 2009, 8, 2952-2964.

(43) Elledge, S. J. Cell cycle checkpoints: preventing an identity crisis. Science 1996, 274, 1664.

(44) Park, K.-J.; Lee, C.-H.; Kim, A.; Jeong, K. J.; Kim, C.-H.; Kim, Y.-S. Death receptors 4 and 5 activate Nox1 NADPH oxidase through riboflavin kinase to induce reactive oxygen species-mediated apoptotic cell death. Journal of Biological Chemistry 2012, 287, 3313-3325.

(45) Camporeale, G.; Zempleni, J. Oxidative folding of interleukin-2 is impaired in flavin-deficient Jurkat cells, causing intracellular accumulation of interleukin- 2 and increased expression of stress response genes. The Journal of nutrition 2003, 133, 668-672.

(46) Pawlowski, J.; Kraft, A. S. Bax-induced apoptotic cell death. Proceedings of the National Academy of Sciences 2000, 97, 529-531.

(47) Oh, Y. S.; Lee, Y. J.; Park, E. Y.; Jun, H. S. Interleukin-6 treatment induces beta-cell apoptosis via STAT-3-mediated nitric oxide production. Diabetes/metabolism research and reviews 2011, 27, 813-819.

(48) Zhang, J.; Zhang, Y.; Dutta, D. J.; Argaw, A. T.; Bonnamain, V.; Seto, J.; Braun, D. A.; Zameer, A.; Hayot, F.; Lòpez, C. B.; Raine, C. S.; John, G. R. Proapoptotic and Antiapoptotic Actions of Stat1 versus Stat3 Underlie Neuroprotective and Immunoregulatory Functions of IL-11. The Journal of Immunology 2011, 187, 1129-1141.

(49) Chou, C.-H.; Chou, A.-K.; Lin, C.-C.; Chen, W.-J.; Wei, C.-C.; Yang, M.-C.; Hsu, C.-M.; Lung, F.-W.; Loh, J.-K.; Howng, S.-L. GSK3 $\beta$ regulates Bcl2L12 and Bcl2L12A anti-apoptosis signaling in glioblastoma and is inhibited by $\mathrm{LiCl}$. Cell Cycle 2012, 11, 532-542.

(50) Sau, A.; Bera, K.; Mondal, P.; Satpati, B.; Basu, S. Distance-Dependent Electron Transfer in Chemically Engineered Carbon Dots. The Journal of Physical Chemistry C 2016, 120, 26630-26636.

(51) Lopez-Acevedo, O.; Akola, J.; Whetten, R L.; Grönbeck, H.; Häkkinen, H. Structure and bonding in the ubiquitous icosahedral metallic gold cluster Au144 (SR) 60. The Journal of Physical Chemistry C 2009, 113, 5035-5038.

(52) Pal, U.; Pramanik, S. K.; Bhattacharya, B.; Banerji, B.; Maiti, N. C. Binding interaction of a gamma. SpringerPlus 2016, 5, 1-17.

(53) Bowers, K. J.; Chow, E.; Xu, H.; Dror, R. O.; Eastwood, M. P.; Gregersen, B. A.; Klepeis, J. L.; Kolossvary, I.; Moraes, M. A.; Sacerdoti, F. D. In Tilte2006; ACM.

(54) Huang, D. W.; Sherman, B. T.; Lempicki, R. A. Bioinformatics enrichment tools: paths toward the comprehensive functional analysis of large gene lists. Nucleic acids research 2008, 37, 1-13.

(55) Thomas, P. D.; Kejariwal, A.; Guo, N.; Mi, H.; Campbell, M. J.; Muruganujan, A.; Lazareva-Ulitsky, B. Applications for protein sequence-function evolution data: mRNA/protein expression analysis and coding SNP scoring tools. Nucleic acids research 2006, 34, W645-W650. 
Table of Content:

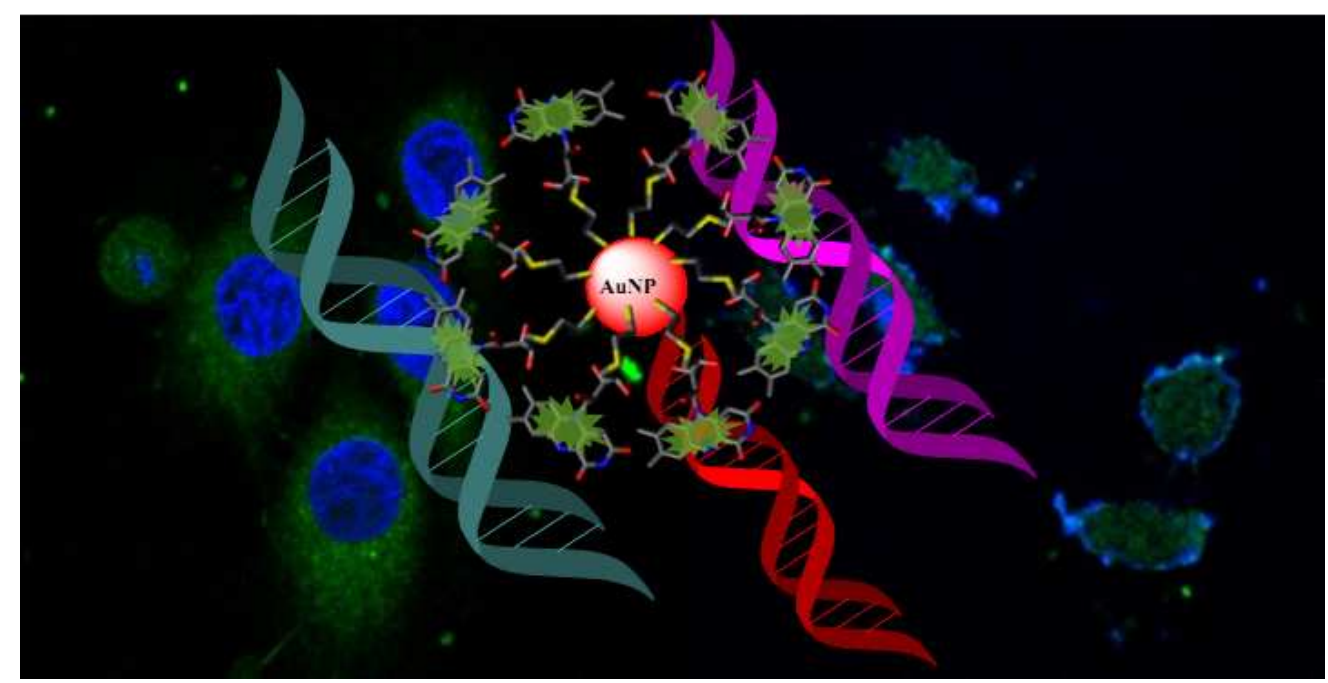

